\begin{tabular}{|c|c|}
\hline Title & 3-D Topology Optimization of Claw-Pole A Iternator Using Gaussian-Basis Function With Global and Local Searches \\
\hline Author(s) & Otomo, Y oshitsugu; Igarashi, Haj ime; Hidaka, Y uki; Komatsu, T aiga; Y amada, Masaki \\
\hline Citation & $\begin{array}{l}\text { IEEE Transactions on Magnetics, 56(1), 1-4 } \\
\text { https://doi.org/10.1109/T MA G.2019.2952205 }\end{array}$ \\
\hline Issue Date & $2020-01$ \\
\hline Doc URL & http:/hdl.handle.net/2115/76840 \\
\hline Rights & $\begin{array}{l}\text { (C) } 2020 \text { IEEE. Personal use of this material is permitted. Permission from IEEE must be obtained for all other uses, in } \\
\text { any current or future media, including reprinting/republishing this material for advertising or promotional purposes, } \\
\text { creating new collective works, for resale or redistribution to servers or lists, or reuse of any copy righted component of } \\
\text { this work in other works. }\end{array}$ \\
\hline Type & article (author version) \\
\hline File Information & Full_Paper_Final_Submission_COMPUMAG.pdf \\
\hline
\end{tabular}

Instructions for use 


\title{
3-D Topology Optimization of Claw-Pole Alternator Using Gaussian-Basis Function with Global and Local Searches
}

\author{
Yoshitsugu Otomo ${ }^{1,2}$, Hajime Igarashi ${ }^{1}$, Member, IEEE, Yuki Hidaka ${ }^{3}$, Taiga Komatsu ${ }^{3}$, and Masaki Yamada ${ }^{3}$ \\ ${ }^{1}$ Graduate School of Information Science and Technology, Hokkaido University, Sapporo, 060-0814, Japan \\ ${ }^{2}$ Research Fellow of Japan Society for the Promotion of Science (JSPS), Tokyo, 102-0083, Japan \\ ${ }^{3}$ Advanced Technology Research and Development Center, Mitsubishi Electric Corporation, Amagasaki, 661-8661, Japan
}

\begin{abstract}
This paper proposes a two-step topology optimization method based on the normalized Gaussian functions. The proposed method is shown effective for design of a claw-pole alternator. In this method, the global search using the micro-genetic algorithm is followed by the local search using the sensitivity analysis based on the adjoint variable method. The three dimensional structure of the rotor is optimized using the proposed method under low and high speed conditions.
\end{abstract}

Index Terms-Adjoint variable method, Claw-pole alternator, Genetic algorithm, NGnet, Topology optimization.

\section{INTRODUCTION}

$\mathrm{R}$ ECENTLY, highly efficient alternators for automobiles have been required because of increase in electric load. A typical shape of the claw-pole alternator which is widely used as a generator for vehicle is shown in Fig. 1. To improve the performance of the claw-pole alternator, the parameter optimization of the rotor core based on field analysis has been carried out [1]-[4]. There are, however, difficulties in the parameter optimization to find novel structures with excellent performance which have never been proposed. On the other hand, the topology optimization in which shape is freely deformed allowing generation and annihilation of holes can lead to novel structures. In particular, the topology optimization based on the normalized Gaussian network (NGnet) has been shown fairly effective for the design of rotating machines [5][7]. In this method, the genetic algorithm (GA) is employed because of its excellent ability in global search. There is weakness in GA, however, that the local search is not well performed for the topology optimization problems with many unknown variables. To overcome this problem, the two-step topology optimization method which combines the on/off method with the level set method has been proposed [8]. In this method, the global search based on the on/off method and GA is followed by the local search based on the level set method. However, this method relies on the complex shape representation using two different basis functions: NGnet for global search and level set function for local search.

In this paper, we propose a novel two-step topology optimization method for 3-D topology optimization, which is effective for design of alternators as well as other machines. In the present method, NGnet is used for both in global and local searches in contrast to the above-mentioned approach. In the

Manuscript received August 17, 2019; revised October 24, 2019; accepted November 4, 2019. Corresponding author: Y. Otomo.

Color versions of one or more of the figures in this paper are available online at http://ieeexplore.ieee.org.

Digital Object Identifier (inserted by IEEE). proposed method, after the global search is performed by GA, the local search is carried out using the sensitivity analysis based on the adjoint variable method. For verification, the proposed method is applied to the $3 \mathrm{D}$ optimization of the rotor core shape of a claw-pole alternator.

\section{TWO-STEP OPTIMIZATION METHOD}

\section{A. NGnet-Based on/off Method with $\mu G A$}

The NGnet-based on/off method is schematically shown in Fig. 2. The material attribute in the design region $\Omega_{\text {core }}$ is determined from the value of the shape function defined by

$$
y(\boldsymbol{x}, \boldsymbol{w})=\sum_{i=1}^{N} w_{i} b_{i}(\boldsymbol{x})
$$

where $w_{i}, \boldsymbol{x}$ and $N$ denote the weighting coefficient, position vector and number of Gaussian functions, respectively, and $\boldsymbol{w}$ is a vector defined by $\boldsymbol{w}=\left[w_{1}, w_{2}, \ldots, w_{N}\right]^{\mathrm{t}}$. Moreover, $b_{i}(\boldsymbol{x})$ is the normalized Gaussian function given by

$$
\begin{aligned}
& b_{i}(\boldsymbol{x})=G_{i}(\boldsymbol{x}) / \sum_{j=1}^{N} G_{j}(\boldsymbol{x}) \\
& G_{i}(\boldsymbol{x})=\frac{1}{(2 \pi)^{\frac{3}{2}} \sigma^{3}} \exp \left\{-\frac{1}{2 \sigma^{2}}\left|\boldsymbol{x}-\boldsymbol{x}_{i}\right|^{2}\right\}
\end{aligned}
$$

where $\sigma$ and $\boldsymbol{x}_{i}$ denote the standard deviation and center of Gaussian basis, respectively. The material attribute $M_{e}$ of finite element $e$ in $\Omega_{\text {core }}$ is determined from

$$
M_{e}=\left\{\begin{array}{cc}
\text { iron, } & y(\boldsymbol{x}, \boldsymbol{w}) \geq 0 \\
\text { air, } & y(\boldsymbol{x}, \boldsymbol{w})<0
\end{array}\right.
$$

It is remarked that the topology optimization is now reduced to the parameter optimization with respect to $\boldsymbol{w}$. In the global search process, $\boldsymbol{w}$ is determined so as to maximize the objective function $F(\boldsymbol{w})$ by the micro genetic algorithm $(\mu \mathrm{GA})$ [9] 
subjected to given constraints. During the optimization, the performance of the alternator is evaluated by solving the finite element (FE) equation of magnetostatic field, $\mathrm{K} \boldsymbol{A}=\boldsymbol{b}$. When $\boldsymbol{w}$ changes, the material distribution as well as $\mathrm{K}$ also change. Hence the field $\boldsymbol{A}$ is the implicit function of $\boldsymbol{w}$.

\section{B. Local Search Method}

Starting from the solution obtained by the global search, the local search based on the sensitivity analysis with respect to $\boldsymbol{w}$ is performed. Since it is difficult to directly evaluate the derivative of $F(\boldsymbol{w})$, we adopt here the adjoint variable method. In this method, $F(\boldsymbol{w})$ is approximated by the modified objective function defined by

$$
\bar{F}(\boldsymbol{w})=F(\boldsymbol{w})+\boldsymbol{\Phi}^{\mathrm{t}}(\mathrm{K} \boldsymbol{A}-\boldsymbol{b})
$$

where $\boldsymbol{\Phi}$ denotes the adjoint variable. Note that $\bar{F} \approx F$ if $\boldsymbol{A}$ is a numerical solution to $\mathrm{K} \boldsymbol{A}=\boldsymbol{b}$. The derivative of $\bar{F}(\boldsymbol{w})$ with respect to $p$-th weighting coefficient $w_{p}$ is given by

$$
\frac{\partial \bar{F}(\boldsymbol{w})}{\partial w_{p}}=\boldsymbol{\Phi}^{\mathrm{t}} \frac{\partial \mathrm{K}}{\partial w_{p}} \boldsymbol{A}+\left[\left(\mathrm{K}+\frac{\partial \mathrm{K}}{\partial \boldsymbol{A}} \boldsymbol{A}\right) \boldsymbol{\Phi}+\frac{\partial F(\boldsymbol{w})}{\partial \boldsymbol{A}}\right]^{\mathrm{t}} \frac{\partial \boldsymbol{A}}{\partial w_{p}}
$$

The second term in (6) can be eliminated by imposing $\boldsymbol{\Phi}$ to satisfy the adjoint equation given by

$$
\left(\mathrm{K}+\frac{\partial \mathrm{K}}{\partial \boldsymbol{A}} \boldsymbol{A}\right) \boldsymbol{\Phi}=-\frac{\partial F(\boldsymbol{w})}{\partial \boldsymbol{A}}
$$

By substituting $\boldsymbol{\Phi}$ into the first term in (6), we can determine the derivative of the objective function. In order to update the material attribute so as to make $F(\boldsymbol{w})$ larger in the optimization, we have to evaluate the derivative of $\partial \mathrm{K} / \partial w_{p}$ from

$$
\frac{\partial \mathrm{K}_{i j}}{\partial w_{p}}=\int_{\Omega_{\text {core }}} \operatorname{rot} \boldsymbol{N}_{i} \frac{\partial v(\boldsymbol{x}, \boldsymbol{w}, \boldsymbol{B})}{\partial w_{p}} \operatorname{rot} \boldsymbol{N}_{j} d \Omega
$$

where $\boldsymbol{N}_{i}, v(\boldsymbol{x}, \boldsymbol{w}, \boldsymbol{B})$ and $\boldsymbol{B}$ denote the vector interpolation function, magnetic reluctivity and magnetic induction, respectively. To evaluate $\partial v(\boldsymbol{x}, \boldsymbol{w}, \boldsymbol{B}) / \partial w_{p}$, the magnetic reluctivity is expressed in the form

$$
v(\boldsymbol{x}, \boldsymbol{w}, \boldsymbol{B})=v_{0}+\frac{v_{\mathrm{m}}(\boldsymbol{B})-v_{0}}{1+\exp \{-\alpha y(\boldsymbol{x}, \boldsymbol{w})\}}
$$

where $\alpha, v_{\mathrm{m}}(\boldsymbol{B})$ and $v_{0}$ denote a constant and the magnetic reluctivity of core with saturation and air, respectively.

\section{OptimizATION PRoblems}

In the optimization, we determine the rotor shape of the 3-D optimization model shown in Fig. 3 (a) so that the electric generation of the claw-pole alternator is maximized. To shape the rotor core, 432 Gaussian functions are uniformly deployed in the design region as shown in Fig. 3 (b), where their distribution is represented by the spheres with radius of the standard deviation. As the first example, we optimize the

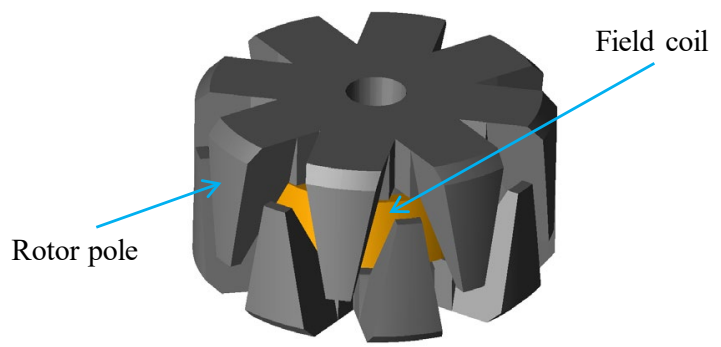

Fig. 1. Convenitonal claw-pole alternator

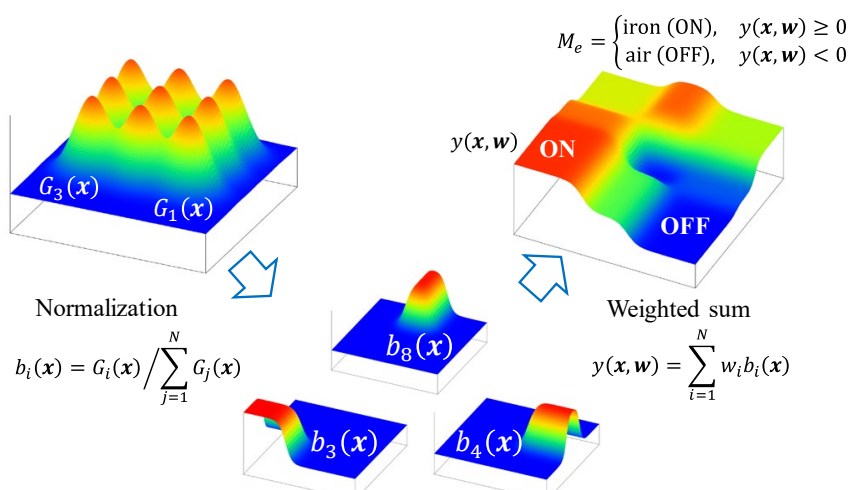

Fig. 2. On/off method using normalized Gaussians (2D example)

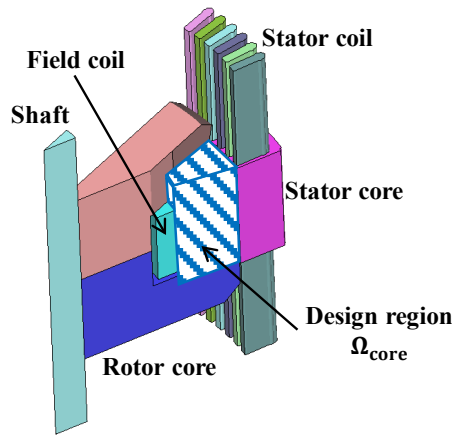

(a) Optimization model (1/16 fraction is shown.)

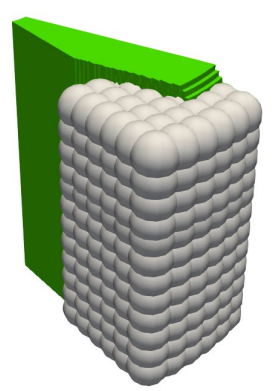

(b) Distribution of Gaussian functions $(\sigma=4.0 \mathrm{~mm})$
Fig. 3. Optimization model and distribution of Gaussian functions

performance of an alternator under low rotational speed condition in which the diamagnetic field generated by the stator currents can be ignored. Because the line voltage between the stator coils, which directly affects the electric generation, is maximized:

$$
\max _{w} F_{1}(w), \quad F_{1}(w)=V_{\mathrm{UV}}^{1000}(\boldsymbol{w})
$$

where $V_{\mathrm{UV}}^{1000}(\boldsymbol{w})$ denotes the open voltage between $\mathrm{U}$ and $\mathrm{V}$ phase coils at $1000 \mathrm{r} / \mathrm{min}$ rotational speed.

In the second example, we consider the influence of the stator currents on the optimization under the high speed condition where the diamagnetic field generated by the stator currents cannot be ignored. The coupling between the alternator with the battery charging circuit would have to be considered for accurate evaluation of the stator currents. Since this strong coupling leads to large computational cost, the stator currents shown in Fig. 3 (a) are assumed to be constant during 
optimization. We found that this remedy is not effective for the topology optimization in [2]. To consider the effect of the stator current and simultaneously avoid the time-consuming coupling analysis, we maximize the total flux due to the rotor and stator currents as follows:

$$
\max _{\boldsymbol{w}} F_{2}(\boldsymbol{w}), \quad F_{2}(\boldsymbol{w})=\Phi_{\mathrm{R}}^{6000}(\boldsymbol{w})-\Phi_{\mathrm{S}}^{6000}(\boldsymbol{w})
$$

where $\Phi_{\mathrm{R}}^{6000}(\boldsymbol{w})$ and $\Phi_{\mathrm{S}}^{6000}(\boldsymbol{w})$ denote the rotor and stator fluxes at $6000 \mathrm{r} / \mathrm{min}$ rotational speed, respectively and minus sign comes from the inverse direction of the magnetic field.

\section{OPTIMIZATION RESUlTS}

\section{A. Optimization Under Low Speed Condition}

To solve optimization problem (10) using $\mu \mathrm{GA}$ for the global search, the number of individuals is set to 5 and the evolution process is continued for 120 generations. Note that random material distributions are generated in the first generation of $\mu \mathrm{GA}$ to obtain an optimal solution which is independent of the initial shape. On the other hand, the local search process after the global search is performed for 120 iterations. In this setting, it takes about three weeks to obtain the optimization results using the Intel Xeon CPU (3.5 GHz, five cores). It is remarked that $70 \%$ of the computing time is spent for the global search.

The optimized results using global search and two-step optimization, represented by "Optimized (Global)" and

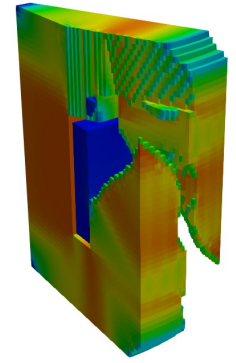

(a) Optimized (Global)

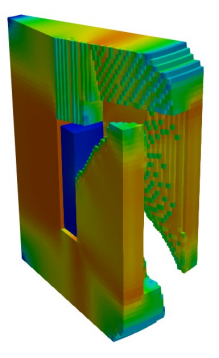

(b) Optimized (Two-step)

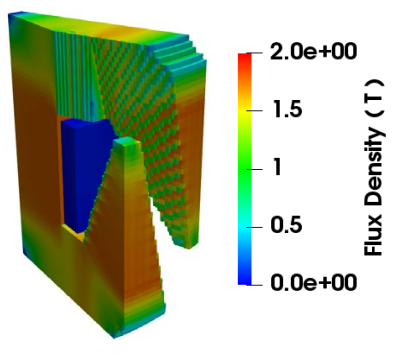

(c) Conventional
Fig. 4. Optimized and conventional rotor core shapes and magnetic induction for low speed condition.

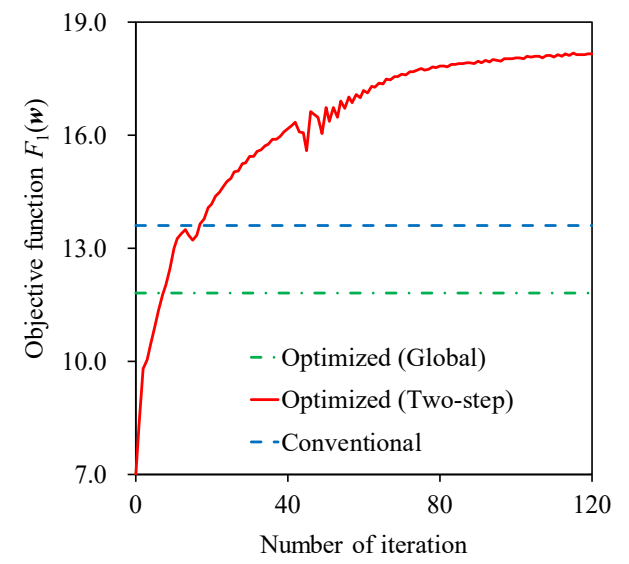

Fig. 5. Convergence history of local search
"Optimized (Two-step)", respectively, are shown in Fig. 4 (a) and (b). For comparison, the conventional rotor shape [2] is shown in Fig. 4 (c). It is evident that the optimized shapes (a) and (b) have different geometrical features in comparison with that in (c). The convergence history of the local search and line voltages for no load condition are plotted in Figs. 5 and 6, respectively. In Fig. 5, the reason why the initial value of the local search is smaller than those of the conventional method

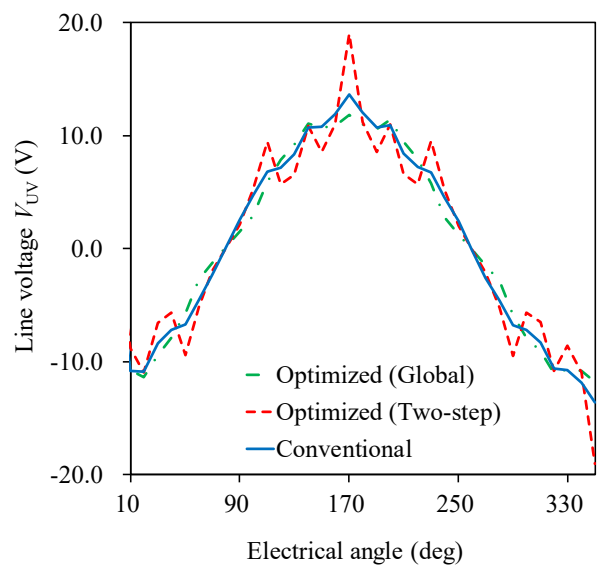

Fig. 6. Comparison results of line voltage without load

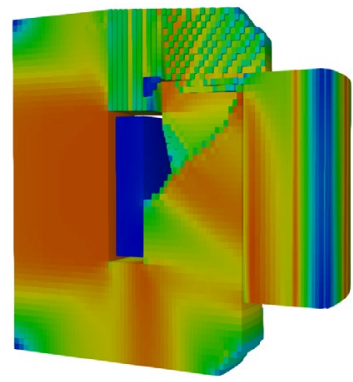

(a) Optimized (Global)

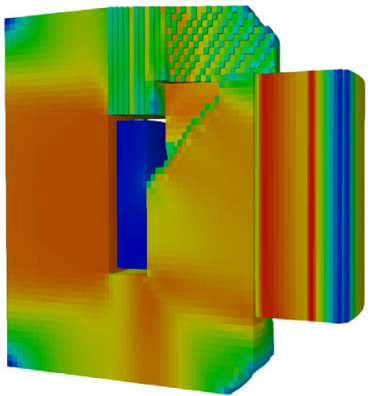

(b) Optimized (Two-step)

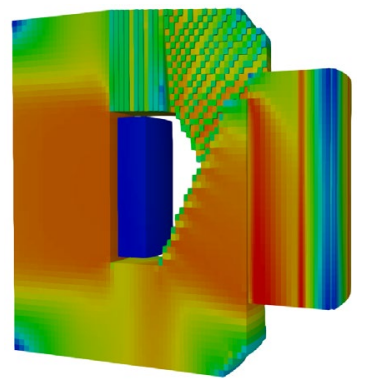

(c) Conventional

Fig. 7. Distribution of magnetic induction in the stator and rotor cores for low speed condition 
and global search is due to the fact that the gray permeability coming from (9) exists in the initial configuration. However, the final value of the local search is much greater than the latter two results. To interpret the different performance among the alternators, the flux density distributions are compared in Fig. 7. It can be seen that the stator core in (b) is strongly saturated over the core because the rotor shape is optimized so that the net interlinkage flux effectively increases. On the other hand, there are non-saturated regions in the stator cores of (a) and (c). In particular, the magnetic flux in the stator of (a) is weaker than that of (c). This result would be due to the weakness of $\mu \mathrm{GA}$ that the local search is not well performed for the topology optimization problems with many unknown variables. It is concluded that the proposed two-step method is effective to increase the induced voltage under the low speed condition.

\section{B. Optimization Under High Speed Condition}

Optimization problem (11) is solved using the proposed method. The distribution of the magnetic induction in the rotor and stator cores considering stator currents are shown in Fig. 8. It can be seen that the surface area of the optimized rotor core increases to effectively cancel the diamagnetic field due to the stator currents. Thus, the magnetic induction of the optimized rotor cores is higher than that of the conventional rotor core. To verify the effectiveness of the optimized rotor shape, we compare the generated currents $I_{\mathrm{DC}}$ shown in Fig. 9. The resultant values of $I_{\mathrm{DC}}$ are shown in the caption of Fig. 8 . We find that $I_{\mathrm{DC}}$ in (a) is much larger than that of (b). It is concluded from these results that the proposed method is effective to improve the performance of a claw-pole alternator.

\section{CONCLUSION}

In this paper, we have proposed a novel two-step topology optimization method based on the NGnet method to effectively improve the performance of a claw-pole alternator. It has been shown that the proposed method works well for low and high speed conditions. The proposed method can also be applied to the topology optimization considering the eddy current loss in the rotor core, which will be discussed elsewhere. In future, we plan to manufacture the optimized rotor to verify its performance by experiments.

\section{ACKNOWLEDGMENT}

This work was supported by JSPS KAKENHI Grant Number JP19J20375, and the MEXT Doctoral program for DataRelated InnoVation Expert Hokkaido University (D-DRIVEHU) program.

\section{REFERENCES}

[1] S. Wu, S. Zuo, and Y. Zhang, "Optimization for Electromagnetic Noise Reduction in Claw Pole Alternator by Rotor Claw Chamfering," IEEE Trans. Ind. Electron., vol. 65, no. 12, pp. 9325-9335, 2018.

[2] Y. Hidaka and H. Igarashi, "Three-Dimensional Shape Optimization of Claw-Pole-Motors," J. Adv. Simulat. Sci. Eng., vol. 4, no. 1, pp. 64-77, 2018.

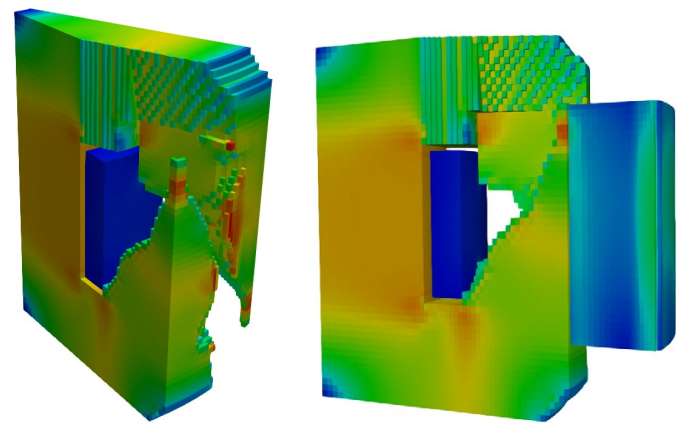

(a) Optimized (Two-step), $I_{\mathrm{DC}}=194 \mathrm{~A}$
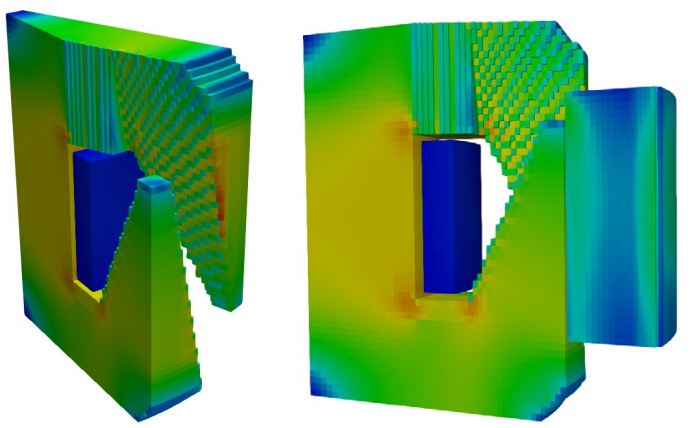

(b) Conventional, $I_{\mathrm{DC}}=176 \mathrm{~A}$

Fig. 8. Distribution of magnetic induction in the stator and rotor cores for high speed condition

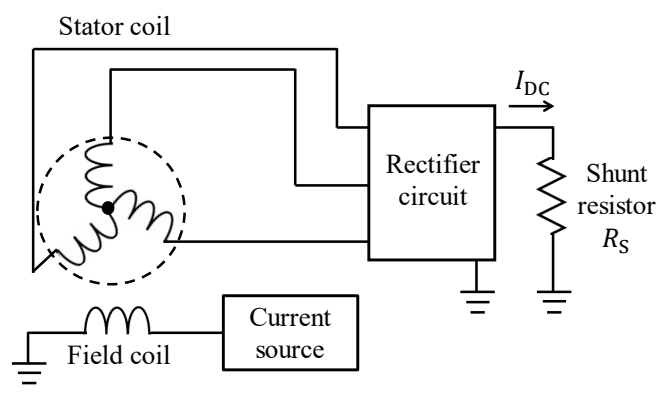

Fig. 9. Power generation circuit

(Rotating speed: 6,000 rpm, Field coil current: $6 \mathrm{~A}, R_{\mathrm{S}}=0.1 \Omega$ )

[3] A. Tan-Kim, V. Lanfranchi, S. Vivier, J. Legranger, and F. Palleschi, "Vibro-Acoustic Simulation and Optimization of a Claw-Pole Alternator," IEEE Trans. Ind. Appl., vol. 52, no. 5, pp. 3878-3885, 2016.

[4] B. Ma, G. Lei, J. Zhu, and Y. Guo, "Design Optimization of a Permanent Magnet Claw Pole Motor with Soft Magnetic Composite Cores," IEEE Trans. Magn., vol. 54, no. 3, Art. no. 8102204, 2018.

[5] T. Sato, K. Watanabe, and H. Igarashi, "Multimaterial Topology Optimization of Electric Machines Based on Normalized Gaussian Network," IEEE Trans. Magn., vol. 51, no. 3, Art. no. 7202604, 2015.

[6] H. Sasaki and H. Igarashi, "Topology Optimization Accelerated by Deep Learning," IEEE Trans. Magn., vol. 55, no. 6, Art. no. 7401305, 2019.

[7] S. Doi, H. Sasaki, and H. Igarashi, "Multi-Objective Topology Optimization of Rotating Machines Using Deep Learning," IEEE Trans. Magn., vol. 55, no. 6, Art. no. 7202605, 2019.

[8] Y. Hidaka, T. Sato, and H. Igarashi, "Topology Optimization Method Based on On-Off Method and Level Set Approach," IEEE Trans. Magn., vol. 50, no. 2, Art. no. 7015204, 2014.

[9] C. A. C. Coello and G. T. Pulido, "A Micro-Genetic Algorithm for Multiobjective Optimization," in Proc. EMO, pp. 126-140, 2001. 